\title{
Optimization of multichip RFID Tag Antenna with Genetic Algorithm and Method of Moments
}

\author{
Yuanhe Xiong, Vecchi Giuseppe \\ Department of Electronics and Telecommunications \\ Politecnico di Torino \\ Torino, Italy
Cecilia Occhiuzzi, Gaetano Marrocco
University of Roma Tor Vergata
Rome, Italy

\author{
Stefano Caizzone \\ Institute of Communications and Navigation \\ German Aerospace Center (DLR) \\ Wessling, Germany \\ Javier Araque Quijano \\ Universidad Nacional de Colombia \\ Bogotá, Colombia
}

\begin{abstract}
-a specific procedure is implemented for the optimization of passive part of multichip RFID tag antenna, based on the performance parameter in terms of newly developed concepts. Examples are given and significant improvements have been observed comparing with previous results, which verifies the approach.
\end{abstract}

\section{INTRODUCTION}

The radiofrequency identification technology (RFID) for object tagging [1] is rapidly evolving toward the "Internet of Things" [2,3]: the convergence of a number of research disciplines (identification, real-time localization, sensor networks, pervasive computing) that enable the Internet to get into the real world of physical objects interacting with web services.

The challenges to the design of present- and nextgeneration RFID have spurred a significant research over the past few years, and the availability of low-cost chips has prompted the study of multi-chip tags.

In this context, electromagnetic (EM) theory has recently re- vamped antenna and radiation concepts into expanding the capabilities of RFID. An interesting such example is the RFID grid paradigm recently appeared [3].

\section{PROTOTYPE OF TAG ANTENNA}

In [3], the concept "grid" is introduced, which represents a generally coupled multitude of UHF tags, including standalone tags in close mutual proximity as well as tags with a multiplicity of embedded RFID microchip transponders able to achieve advanced capabilities such as redundancy and sensing; also it's fully investigated as an EM interconnected system. In our work, we take the latter formation of grid, i.e., a tag with multiple chips connected to it; this tag can be handled as a multi-port antenna. One prototype of the antenna is shown in Fig. 1, which is derived from T-match dipole; the blue and green regions are both metal.
The passive part (metallization) of the multi-chip tag is automatically designed to optimize the global parameters introduced and discussed in [3], rather than individual radiation or (multi-port) impedance matching, which is a novel approach. The global parameter is named embedded realized gain and it closely integrates parameters like impedance matching, gain and polarization factor into a single parameter. It's of great importance when investigating the reading range of tag antennas.

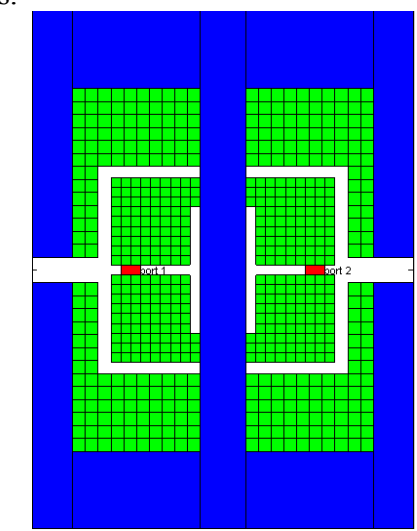

Figure 1. Tag antenna prototype.

\section{OPTIMIZATION IMPLEMENTATION}

Recent embodiments of multi-chip and grid types of tags have shown the necessity of optimizing the EM properties of the passive part of the tag, also for performance robustness. Along this line, we have undertaken an effort to design this passive part via automatic optimization, yet keeping the information on the chip(s) directly in the process (linear behavior considered, as usual). We employ in our contribution the approach devised in [4], which explored the combination of genetic algorithm (GA) and method of moments (MoM). Some techniques were developed to reduce the computational burden of the code, saving both time and memory. This approach is 
especially useful for electrically small antennas, and since it considered multi-port structures, reconfigurability is well suited for the present endeavor.

Consistency checks against previous partial optimization of tags [5] haven been preliminarily carried out. The results are obtained by automatic optimization and presented in detailed structure, and efforts were done to keep complexity within fabrication process requirement. Considering the fact that in our work output structures are usually non-intuitive, which brings inconvenience for fabrication, structural complexity reduction was also considered within and after the optimization, while keeping in mind that the performance of the output structures should not degrade too much. One output structure was fabricated and measured; comparisons were then made between the measured and simulated results to verify the feasibility of our approach.

\section{OPTIMIZATION EXAMPLE}

Reference [5] proposed a two-port tag antenna for detection of thermal threshold with the help of a shape-memory alloy, which was done via sweeping a set of antenna dimension parameters. Based on the information that the reader collects from the tag antenna, decision on whether the temperature goes beyond the threshold will be made. In our work, the application of GA enables an automatic optimization, while the way we divide the prototype enriches the search space. The objective function in our work, which plays a key role in GA, is inherited from [5], which one can refer to for more detailed information.

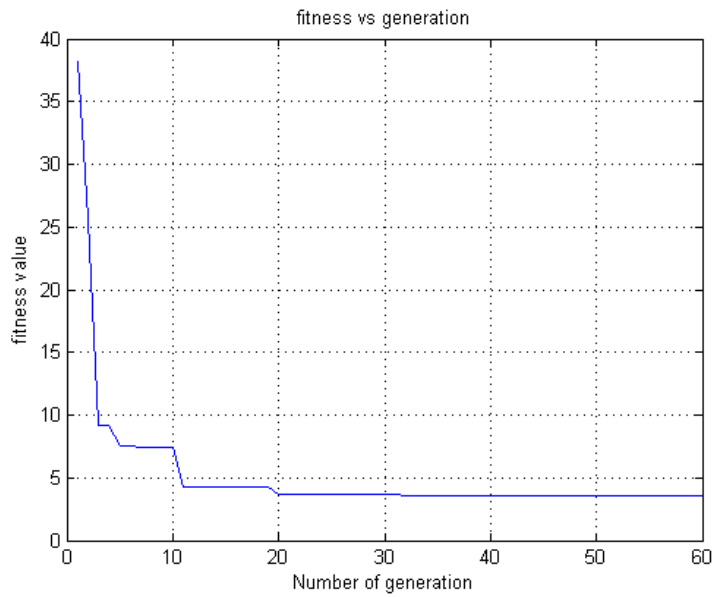

Figure 2. Convergence of fitness value versus generation number

The fitness value for the structure in [5] is around 20, after computing explicitly its EM parameters, while optimizer can easily generate individuals that show fitness value around 3 , the improvement is obviously magnificent. The fitness-versusgeneration curve is shown in Fig. 2 [6]. One output structure is presented in Fig. 3.

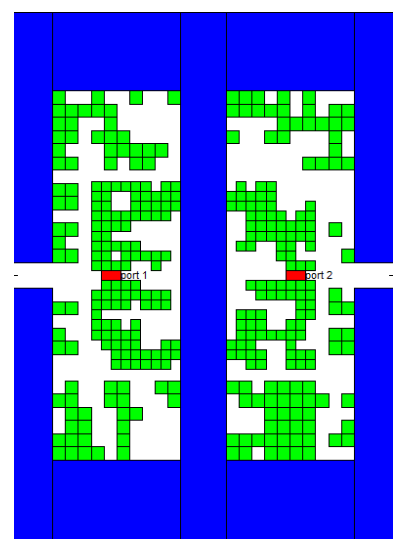

Figure 3. Example of an output structure

\section{CONCLUSION}

Multichip RFID tag antenna optimization problem has been addressed in our contribution, based on the newly developed concept. An example is given and structure performance improvement is reported, which verifies the feasibility of our approach. The approach can be extended further to more complicated problems like 4-port antennas, which will be the focus of our future work.

\section{ACKNOWLEDGEMENT}

This work is sponsored by Italian MIUR Program PRIN 2008 through the grant 2008CRSHTE 004 (MULTI-TAG: Electromagnetic technology for multiple-interrogation active and passive RFID systems).

\section{REFERENCES}

[1] D. M. Dobkin, "The RF in RFID: Passive UHF RFID in Practice." Amsterdam: Elsevier, 2007.

[2] D. Preuveneers and Y. Berbers, "Internet of Things: A contextawareness perspective," in The Internet of Things: From RFID to the Next-Generation Pervasive Networked Systems, L. Yan, Y. Zhang, L. T. Yang, and H. Ning, Eds. London: Auerbach Publications, 2008.

[3] G. Marrocco "RFID Grids: Part I-Electromagnetic Theory", IEEE Transactions on Antennas and Propagation, vol. 59, No. 3, MARCH 20111019.

[4] J. L. Araque Quijano and G. Vecchi, "Optimization of an innovative type of compact frequency-reconfigurable antenna," IEEE Transactions on Antennas and Propagation, vol. 57, pp. 9-18, Jan. 2009.

[5] S. Caizzone, C. Occhiuzzi and G. Marrocco, "Multi-Chip RFID Antenna Integrating Shape-Memory Alloys for Detection of Thermal Thresholds", IEEE Transactions on Antennas and Propagation, vol. 59, 2011.

[6] Yuanhe Xiong, S. Caizzone, C. Occhiuzzi, G. Marrocco, Vecchi G. and Orefice M, "Automatic Optimization of Multichip RFID Tags", 2012 Fourth International EURASIP Workshop on, pp. 128-131, Sep. 2012. 